\title{
IMPLEMENTATION AND CONTROL OF INTEGRAL CYCLE CONTROLLER FOR RESISTANCE SPOT WELDING
}

\author{
N.D. Mehta ${ }^{1}$, D. K. Bhatt ${ }^{2}$ \\ ${ }^{1}$ Assistant professor in Power Electronics Department at VGEC, Chandkheda Ahemadabad \\ ndmehta@vgecg.ac.in and devangkbhatt@yahoo.com \\ ${ }^{2}$ Assistant Professor, Electrical Engineering Department of VGEC, Chandkheda Ahemadabad since 2010.
}

\begin{abstract}
:
The Resistance Spot Welding (RSW) process requires simultaneous application of high electrical current and appropriate pressure over short periods in order to keep the molten material inside the weld zone. Values of these parameters vary in function of the characteristics of the materials to be welded. The application of the electrical current is by means of two copper electrodes brought in contact with the welded parts while the required pressure is applied by means of pneumatic or hydraulic systems. This paper presents the simulation, design, and control of resistance welding system which is used for performing a welding process. The paper is discussed about two aspects. First, simulation of the concept of ICC used for the specific application of RSW. Secondly, the critical factor and method employed for the implementation of the controller. This paper also discuss about the implementation methodology of the RSW. The simulation for the RSW is carried out in PROTEUS software.
\end{abstract}

Index Word: Constant Current Feedback, Integral Cycle Control (ICC), Resistance Spot Welding, Sequential Timer. $* * *$

\section{INTRODUCTION}

In the literature, authors have attempted to control the nonlinear power control system for the RSW to be fully linearised system by applying the non linear feedback linerised technique based on different geometry theories [1]. An intelligent control based on fuzzy logic was developed [2] and addresses the operating condition detection and nugget growth estimations [3]. The variation of internal forces during the process of RSW is also published [4]. Microcontroller based [5] and single chip microcomputer for the intelligent control of the constant current spot welding inverter based on fuzzy control [6] also demonstrated.

All these methods involving either phase control technique or welding inverters. In this paper author described Integral Cycle Control (ICC) for the application of resistance spot welding .The power supply for RSW, methods of controlling, critical factor in RSW and principle of RSW is published in [7]. In this paper the details of implementation and control is described including necessary simulation.

\section{CONCEPT OF INTEGRAL CYCLE CONTROL}

Integral cycle control (ICC) is used for controlling power to an ac load by permitting few full cycles to power the load followed by off period. This is repeated cyclically. The duty cycle is controlled for changing the output power basically it is an on - off control similar to the obtained through thermostatic switches except that here an integral number of cycle are passed. In literature, ICC is also described as On Off control, Burst firing, Zero Voltage Switching (ZVS), Cycle Selection and Cycle Syncopation [4]. Fig. 1 shows the output voltage waveform of Integral cycle controller. When the power is $\mathrm{ON}$, during $\mathrm{N}$ cycle the speed or temperature increases exponentially from a minimum value and reaches a maximum at the end of the $\mathrm{N}^{\text {th }}$ cycle. If $\mathrm{N}$ us the number of full cycles passed per $\mathrm{M}$ cycles of the source voltage then it is said to have a duty cycle of $\mathrm{D}=\mathrm{N} / \mathrm{M}$. The difference between maximum of temperature and the minimum temperature is called the differential.

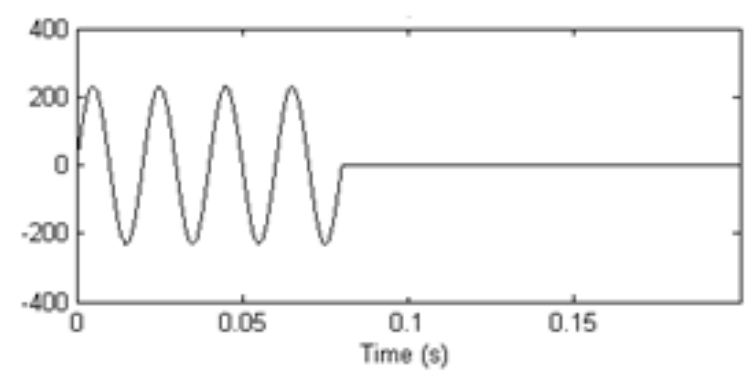

Fig.1 Output of Integral Cycle Controller

If $\mathrm{M}$ is increased their differential increases and vice versa. Low value of $\mathrm{M}$ limit the variation in the value of $\mathrm{N}$, as the smallest power available is

$$
\begin{array}{lc}
\mathrm{P}_{\min }=\left(\mathrm{Vs}^{2 *} 1\right) / \mathrm{M} * \mathrm{R} & \text { when } \mathrm{N}=1 \\
\mathrm{P}_{\max }=\mathrm{Vs}^{2} / \mathrm{R} & \text { when } \mathrm{N}=\mathrm{M} \\
\text { Control Ratio }(\mathrm{CR}) \quad=\quad \mathrm{P}_{\max } / \mathrm{P}_{\text {min }}=\mathrm{M}
\end{array}
$$

The CR is independent of $\mathrm{N}$ but depends on $\mathrm{M}$. ICC gives a much higher differential than phase control. Therefore it can 
be applied only to those processes where the process time constant is large, which keeps the differential low and higher value of $\mathrm{M}$ can be Chosen. Average power consumed by the load can be varied by Changing $\mathrm{N}$ keeping $\mathrm{M}$ fixed, Changing $\mathrm{M}$ keeping $\mathrm{N}$ fixed or changing both. Fig. 2 shows the variation of power and power factor for an ICC in terms of controlled load.

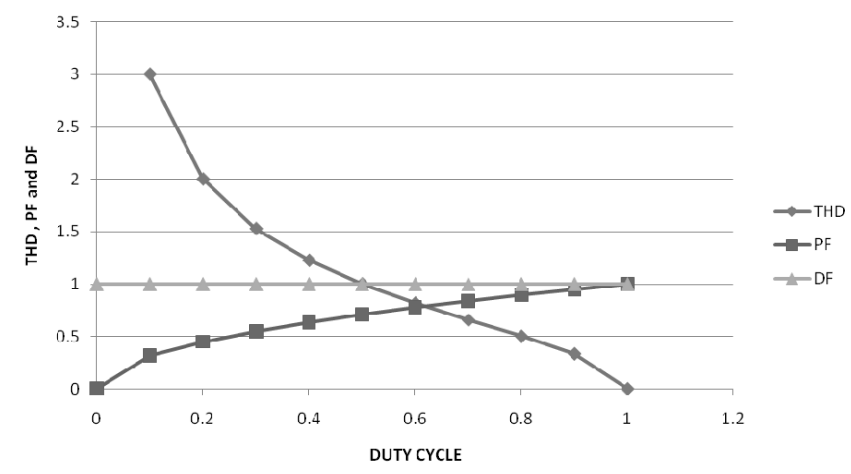

Fig. 2 Variation of RMS value and PF

\section{PERFORMANCE CHARATERISTIC}

SSPC is modeled as an ac voltage source or current source connected to a load through a network of switches. Switches including diode are assumed to be ideal and unidirectional, zero on state losses and unrestricted voltage or current carrying capabilities for a simplified analysis and the criteria for measuring the performance of the converter operation. The steady state converter performance measures can be put into four categories: Voltage Transfer Ratio (VTR), Current Reflection Ration (CRR), harmonic profile, component stresses.

VTR: The forward voltage transfer ratio is defined as the ratio of the rms voltage output voltage to the rms input voltage.

CRR: The reflective characteristic gives the effect of load current on the current drawn by from the source. The CRR is defined as the ratio of the input current to the rms load current.

Harmonic Profile: The output voltage and source current wave form are non sinusoidal. The lowest undesired harmonic frequency in the voltage and input power factor are the measures used for the undesirable Fourier Component.

Component Stresses: Voltage and current in the switch may consist of high transient which may be considering higher than the normal operating voltage. These transients voltage dictate the selection of switching devices and hence determine the cost. The component stress is measured as the ratio of the peak Voltage (Current) to the rms value of the components.

\section{ELECTRONIC CONTROL USED IN RSW}

The following are the list of important Electronic Welding controls generally employed in the RW. [7]
* Line Contactor: This line contactor connect the welding transformer primary to the a.c. supply for interval controlled by the control signals from control circuits. An electronic line contactor uses two SCRs back to back. This method uses very small amount of the control current to control large current.

* Heat Control: This is an electronic circuit which delays the firing of the SCR in the line contactor by a specific angle in each half cycle of the voltage and thus controls the amount of heat given to the held. If the firing angle is increased, less current flows and less amount of the heat is generated. The firing angle can be controlled by any suitable method.

* Non - Synchronous Weld Timer: This is an electronic circuit which governs the number of cycles for which the line contactor remains closed. In can control conduction to the nearest integral number of cycles. However, the angle of firing in the first half cycle is arbitrary and variable in this simple electronic timing circuit.

* Synchronous Weld Timer: This is more elaborate electronic timing circuit which not only control full cycles of conduction accurately but also controls the firing of the line contactor precisely at the desired angle in each cycle so as to avoid production of undesired welding current transients.

* The Sequence Timer: This is more elaborate electronic circuit which includes :

Squeeze time - It is the time that elapsed between the electrode pressure first applied on the work and the initial application on the current. Weld time - It is the time for which the welding current, expressed in the cycle, actually flows to melt the metal at the interface.Hold Time - It is the time during which the pressure is kept applied at the point of the welding after the welding current has ceased to flow. Off Time - After the weld is perfectly hardened, the time that elapses before the electrodes reclose, if the welding machine is still energized, is OFF time. [7]

* Pulsation Timer: This is a timer which controls the number of cycles for which the welding current flows and the number of cycles for which it is allowed. It is a variation of spot welding. In pulsation welding, the welding current flows intermediately unlike spot welding in which current flows continuously until the weld is formed. This intermittent flow of current permits the water cooled electrodes to remove heat from the surfaces of the materials and thereby cause higher temperature gradient near the weld. This method is popularly used for pieces thicker than $1 \mathrm{~cm}$.

\section{SEQUENTIAL TIMER}

Fig. 3 explains the electronic circuit used for sequence timer. Assume that the repeat non-repeat switch (S) is in the non-repeat position (open). When the foot-operated switch or initiation switch is depressed, current flows through the path $(\mathrm{a} 0$ - a1- a2 - RELAY1 - 4a- b1- b0) and relay 1 energizes, as the hold relay is off. Normally open contacts $1 \mathrm{a}, 1 \mathrm{~b}$ and $1 \mathrm{c}$ are closed, the solenoid valve is energized. 
Energization of the solenoid valve allows air to be admitted into the upper chamber of a double acting cylinder of the welding machine. The intakes of air into the cylinder bring down the electrode against the work piece and the necessary pressure is built up thus squeeze operation starts. As contacts $1 \mathrm{~b}$ closed which are in parallel to the foot switch, relay 1 continues to energize even when the foot is removed from the switch. Contacts $1 \mathrm{c}$ are closed which permit the squeeze relay to energize via the path $(\mathrm{a} 0$ - a1- a2- $\mathrm{a} 3$ SQUEEZE RELAY 2 - 1c - 3a- 4b-b3-1b-b1-b0) path, when the squeeze time is over.

One should note here the difference between the statements that the relay circuit is energized and the relay is energized. Actually, if the relay circuit is energized at time $t=0$, then the relay energizes after the time delay SQUEEZE RELAY 2 passes.

When the squeeze time is over, squeeze relay 2 energizes, as the weld relay and hold relay are off. Contacts $2 \mathrm{a}$ and $2 \mathrm{~b}$ are closed via the path (a0- a1- a2 - a3 - a4 - WELD RELAY 3 $-2 b-4 b-b 3-1 b-b 1-b o)$ path. The welding current now passes through the work material as contact $2 \mathrm{a}$ starts the electronic line contactor and welding process starts. Contacts $2 b$ when closed permit the weld relay to operate. Weld relay energizes when the weld time is over. This opens contacts $3 \mathrm{a}$ and closes contacts $3 \mathrm{~b}$ and $3 \mathrm{c}$.. When contacts $3 \mathrm{a}$ are opened, Squeeze relay is de - energized, so contacts $2 \mathrm{a}$ and $2 \mathrm{~b}$ are opened. Due to contacts $2 \mathrm{a}$ being opened Welding current stops. Opening of contacts $2 b$ will no de energizes the weld relay as the contacts $3 \mathrm{~b}$ are in parallel to the contacts $2 \mathrm{~b}$, so weld relay continues to energize through $3 \mathrm{~b}$. Closing of contacts $3 \mathrm{c}$ permits hold relay to energize.

Weld the hold time is over, the hold relay 4 is energized as off relay 5 is off via $(\mathrm{a} 0-\mathrm{a} 1-\mathrm{a} 2-\mathrm{a} 3-\mathrm{a} 4-\mathrm{a} 5-$ HOLD RELAY 4 - 5a - 3c - b5-b4 - b3 - 1b- b1- b0) path. Contacts $4 \mathrm{a}$ and $4 \mathrm{~b}$ are opened the contacts $4 \mathrm{c}$ and $4 \mathrm{~d}$ are closed. When contacts $4 \mathrm{a}$ are opened, relay $11 \mathrm{~b}$ and $1 \mathrm{c}$ are opened to restore the original circuit condition.

If the operator has lifted the foot from the switch, opening of contacts $1 \mathrm{~b}$ will de - energize the relay.

However by chance if the foot is continued to press the switch hold relay will not be de - energized and welding operation cannot repeat as the contacts $4 \mathrm{a}$ and $4 \mathrm{~b}$ are in open condition. To repeat the process, the operator has to remove the foot and depress the switch again. Now suppose the switch is in the repeat position, In this case when the hold relay is energized, contacts $4 \mathrm{~d}$ are closed. When the off time is over, off relay 5 is energized, the contacts $5 \mathrm{a}$ are opened making hold relay to off. If the foot switch is continued to be depressed, the process will repeat as contacts $4 \mathrm{a}$ and $4 \mathrm{~b}$ are closed again due to de emerging of hold relay.

Thus in non-repeat operation, if the foot - switch is continuously depressed, operation is not repeated, where as in repeat operation, the foot - switch is required to be depressed continuously to get repeat condition.

\section{IMPLEMENTATION OF POWER CIRCUIT}

Power circuit of a full wave integral cycle controller for spot welder is given in Fig.4. It uses two SCRs, connected in anti parallel and controls the full wave ac voltage impressed on to the primary winding of the welder transformer. The application requirement is conduction of even number of half cycles on to the transformer to avoid static magnetization. Second requirement is a phase delay in the first half cycle equal to the effective power factor of the load to avoid current transients. Third requirement is of adjustment of number of integral cycles passed on to the transformer during the weld. The circuit involving the OPAMP as ZCD circuit and UJT driver circuit for the triggering the SCR. The back to back SCR is used along with the welding transformer for the proposed RSW. For the simulation of the power circuit PSIM and PROTEUS software is used.

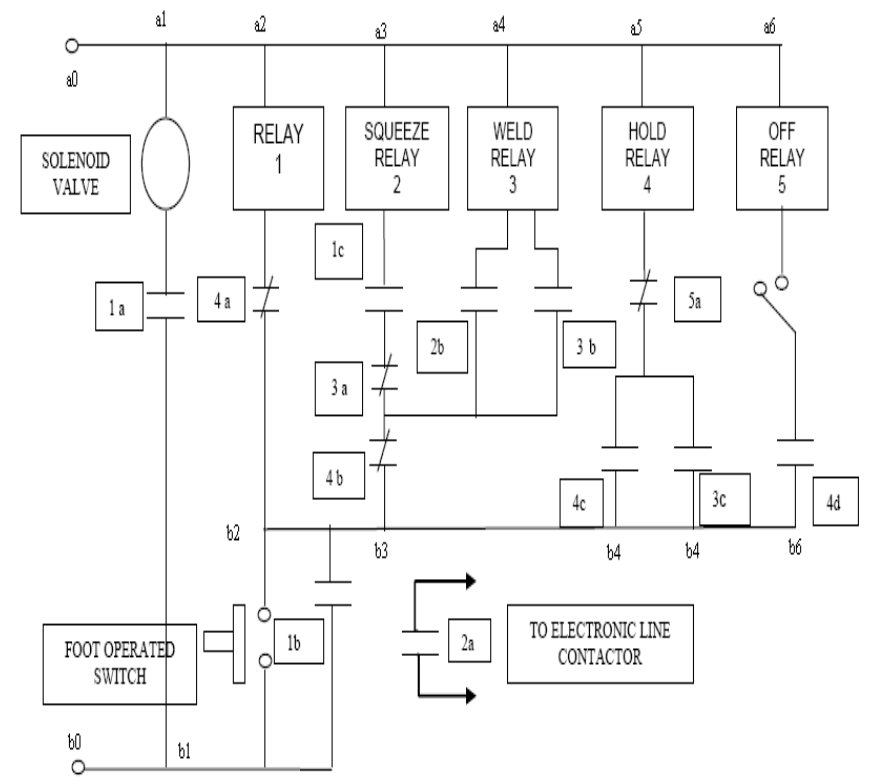

Fig. 3 Power circuit of a full wave ICC for RSW.

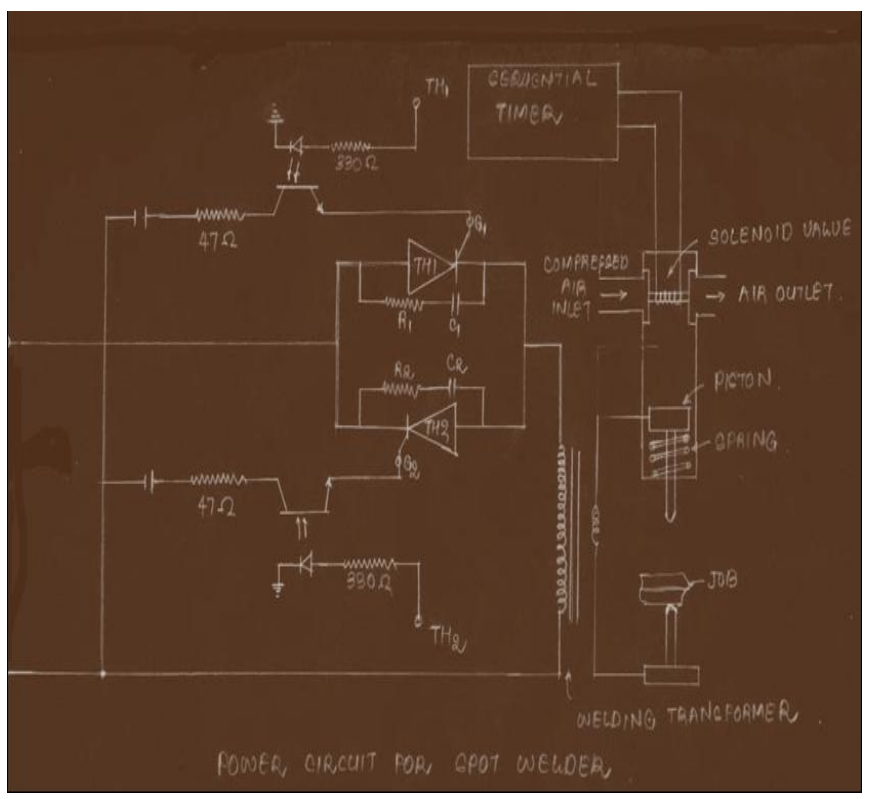

Fig. 4 Electrical Control Circuit for Sequence Timer 
Fig. 5 shows the photograph for the control circuit used for the RSW. The simulation is carried out in the PROTEUS software and waveform of the control circuit for RSW is explained in Fig. 7(b).

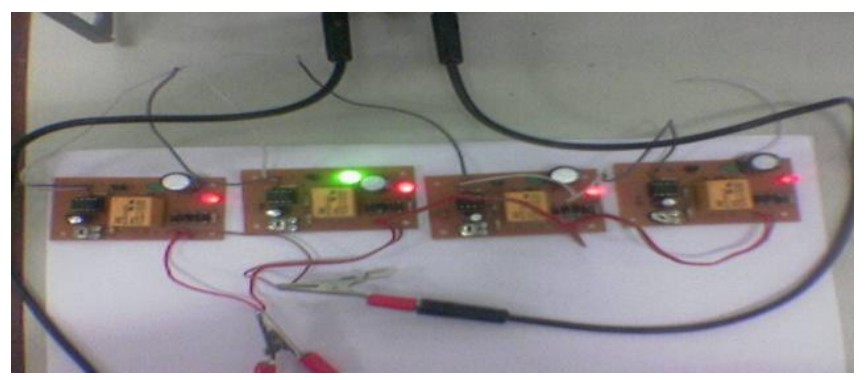

Fig. 5 Photograph for the timer circuit used for RSW.

In the control circuit is a square wave generated by the weld timer immediately after the squeeze period is applied. Two zero crossing detector $\mathrm{ZCD}_{1}$ and $\mathrm{ZCD}_{2}$ produce pulses at positive and negative, zero crossing of the single phase input supply respectively. The positive zero crossing pulses are applied to the UJT firing circuit for the $\mathrm{TH} 1$ output of the triggers a power semiconductor device, in this case SCR 1 .

\section{APPLICATIONS}

This SSPC is essentially a variable duty cycle switch with zero crossover switching. Unlike normal power controller which operates on the principle of variable phase angle Triggering, this circuit switches on power to lad nearly zero crossover points. This controller eminently suited to control of the resistive loads having large thermal hysteresis, e.g. heaters, toaster, soldering irons, oven, cloth iron etc. Inductive loads cannot be controlled. This method can be modified for the inductive loads even and sophisticated display should be incorporated which displays quantities like current, voltage, duty cycle etc. Higher load can also be controlled by using a triac with larger current rating with suitable heat sink provided. Back to back thyristor pair is also used for the even high power rating. ICC can be used in applications where the process time constant is very large compared to $\mathrm{M} * \mathrm{t} \mathrm{i}$, that is the reason it is very popularly used in resistance spot welders where $\mathrm{M}$ is large and it results in good quality welding with uniformity for all jobs. Fig. 6 shows the photograph hardware fabricated for the SSPC for using ICC.

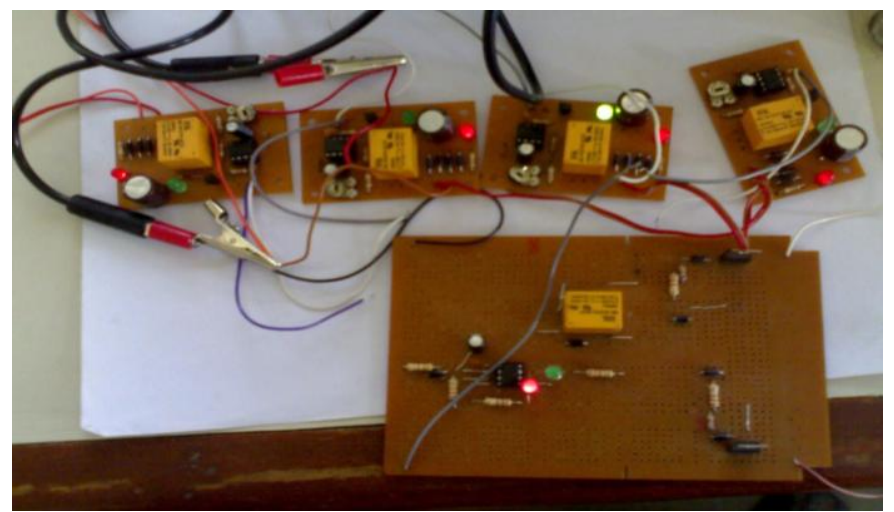

Fig.6Photograph of Control Hardware for the RSW.

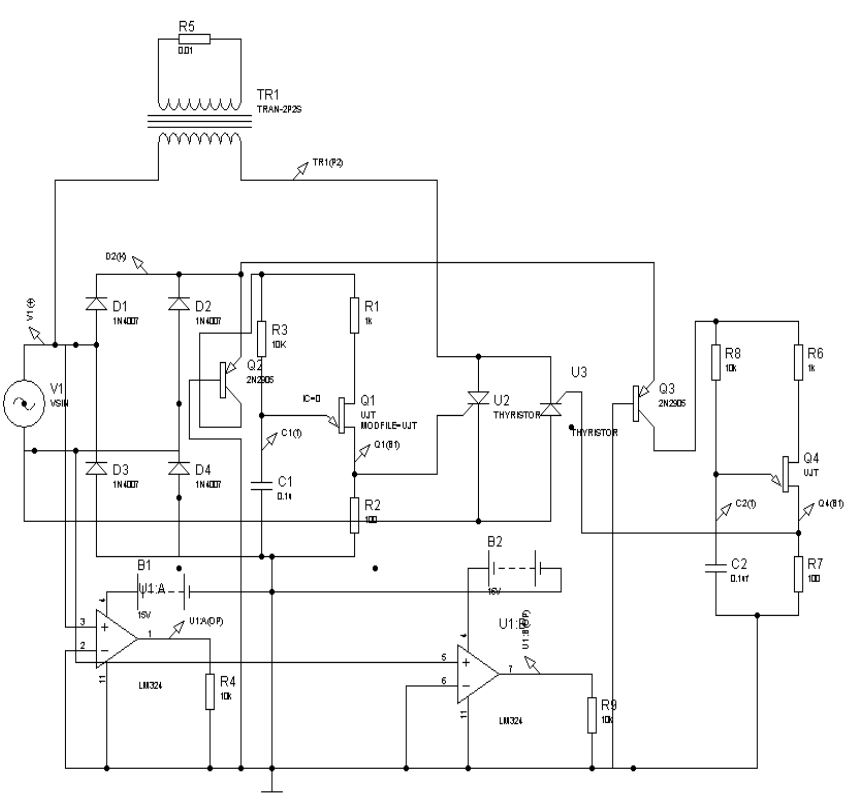

Fig. 7 (a) Circuit Diagram of the Control circuit of RSW

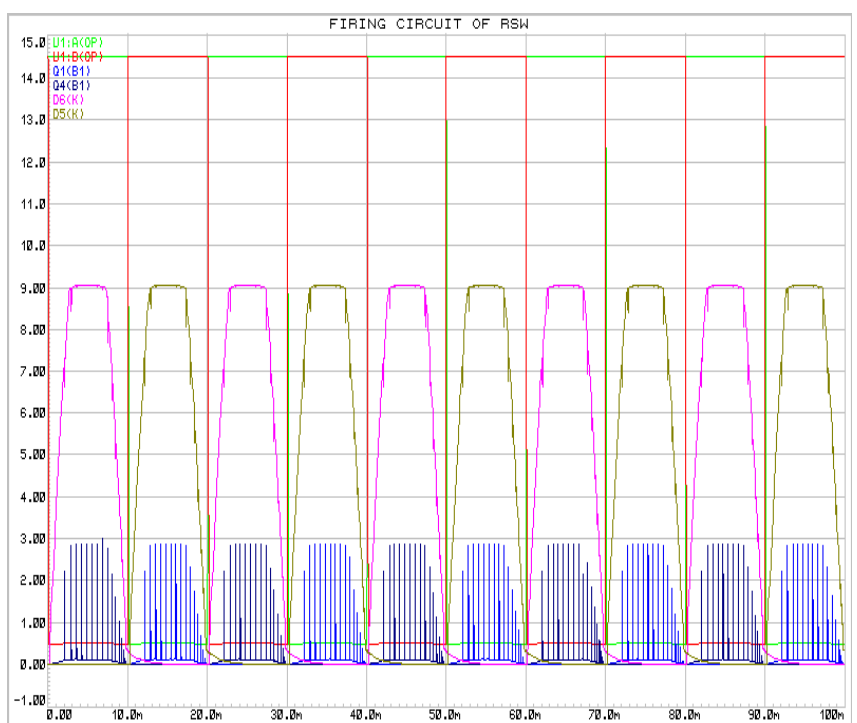

Fig. 7 (b) Control circuit waveform of the Resistance Spot welding

\section{CONCLUSION}

Low cost ICC has been simulated, design and implemented for the resistance spot welding control. As discussed in the different section the harmonic profile of the ICC is improved than PCS. In case of heat controllers of resistive load (R), PCS cause higher order harmonics and generate Radio Frequency Interference (RFI) and heavy inrush currents while switching on from cold. At large power levels, it requires bulky and expensive line filters to minimize RFI. These problems easily overcome by using ICC for heating application. Despite of these advantages, ICC suffering disadvantage of problem of inter harmonic and only suitable to heavy machines and heating systems that have high thermal time constants may reduces the uses of this method. Control circuit is somewhat complex but ease in construction 
and low hardware cost. The fabrication of the RSW is carried out as per simulated in PROTEUS software.

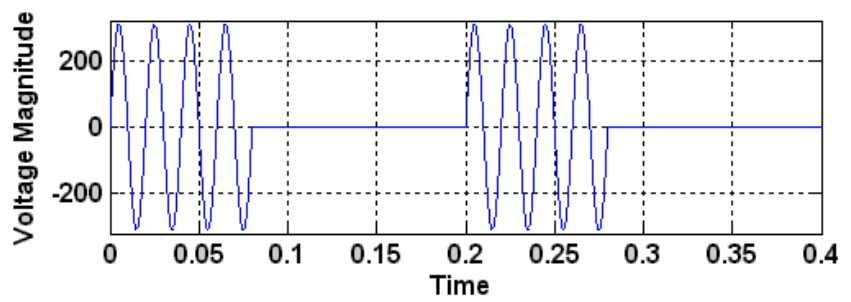

Fig. 8 (b) Otput Load Waveform of ICC D = 0.7

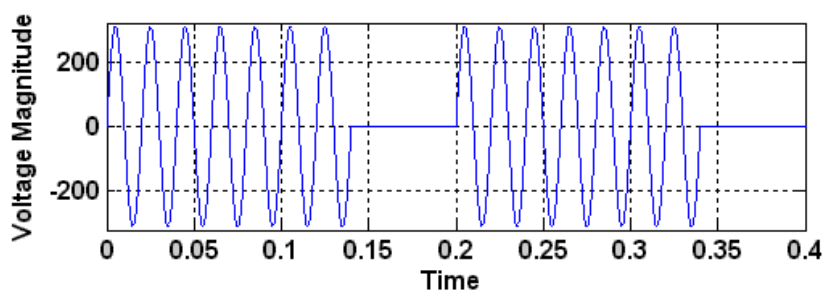

Fig. 8 (a) Output Load Waveform of ICC $D=0.4$

Fig. 8 (a) and Fig. 8 (b) show the output load waveform for ICC for duty cycle 0.4 and 0.7 respectively. The power factor is low for the low duty cycle (small value of D), hence ICC is used for the control only without worrying about the efficiency.

\section{REFERENCE}

[1].Jun Seo-Moon, Gyu-Sik Kim, "Power Control of Resistance Spot Welding System with High Dynamic Performance" IEEE Journal of Ind. Elect. Vol. 2, pp 845-849. Nov 1997

[2].Robert W. Messler, Jr., Min Jou and C. James Li "An Intelligent Control System For Resistance Spot Welding Using a Neural Network and Fuzzy Logic, IEEE Trans. Ind. App. Conf. Vol. 2 pp. 1757-1763 Oct 1995.

[3].David Mintz, John T. Wen "Process Monitoring and Control for Robotic Resistive Spot Welding" $4^{\text {th }}$ IEEE conference on Control Application, Vol. 3 pp. 1126-27 July 1995

[4].H Tang, W Hou and $\mathrm{S} \mathrm{J} \mathrm{Hu}$ "Forging force in resistancespot welding" Proc Instn Mech Engrs Vol 216 Part B: J Engineering Manufacture July 2002 pp 957 968 .

[5].T.C. Manjunath , S. Janardhanan and N.S. Kubal "Simulation, Design, Implementation and Control Welding Process Using Micro - controller" IEEE $5^{\text {th }}$ Asian Control Conference, Vol. 2 pp. 828 -836 Aug 2004.

[6].Shu, Jiang Li, Cheng. Jun Lin, Yun Long Chang "Intelligent Control of Spot Welding Inverter Based on Single Chip Microcomputer" International Conference on Ind. Elect. and Appl. June 2006

[7].N.D.Mehta , B.B.Kadam "Design and Simulation of Integral Switching Cycle Control for Resistance Spot Welding " proceeding of ICETATES 2008 vol.2 pp.1531-42, JAN 2008. 